\title{
The Visualization Research of Villus on Plant Organ Surface Yingying Yin ${ }^{1, a}$ \\ ${ }^{1}$ Jilin Agriculture University, Changchun, China, 130118
}

Keywords: simulation of villus, virtual plant, visualization

\begin{abstract}
For the aim that implement the visualization of plant villus vividly and effectively, do much work on the research of villus outward appearance, then put forward a method to simulate villus on computer, the method adopt segment to construct a single villus, and then, with the addition of parameters such as density, direction, length and radium, villus on plant surface simulation model is completed. The vivid simulation result proved that this model is suitable to simulate villus on plant surface.
\end{abstract}

\section{Introduction}

On the surface on plant organs, such as stem, leaf, branch, fruit, petal and so on, there are always a thin layer of villus, the form of them are different from each other. In the area of computer graphics, the modeling and drawing of trichomes is an important research issue, but, now, most research are all about human's or animal's hair[1], the research about plant villus is fewer and fewer[2]. This paper put forward a method to visualize the single villus base on elements, and, use a serious of parameters to control the pattern of villus on plant surface.

\section{The geometric representation of villus}

There are many methods to draw villus, such as curve[3], segments, cylinder, triangular facets[4], and so on. In this paper, we adopt segments and cylinder to draw a single villus, it use a serious vertex to store a single villus, these vertex is the control points of B-spline curves at the same time, and the B-spline curves generate segments or cylinder, splicing them, a single villus is ok.

Because villus are all very slim, so, using segments to model them is very suitable[5], the numbers of segments to splice a single villus is decided by its bend degree. Another problem is that the shape and size of villus are all different, so it is impossible to adjust their form one by one[6]. A solving method is make several individual villus as different forms, when putting villus on plant surface, choosing one randomly. The individual villus are shown in Fig.1.

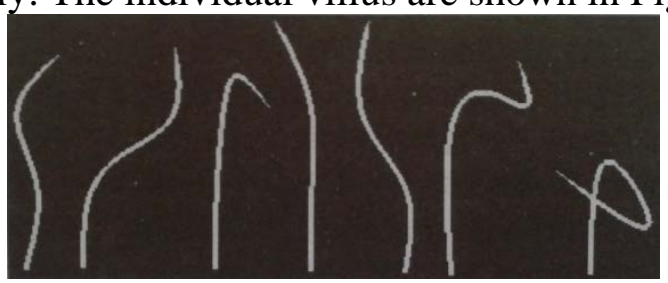

Fig.1 Different individual villus

\section{Parameter controlled}

The villus shape and outward appearance are difference from each other, so, adjust every villus shape is impossible, then, we can define a series of parameter to control length, radium, direction and other properties. In this way, the model can be used to simulate various sorts villus through adjusting parameters.

\section{Villus density}

If adopt mapping function based on preparatory computation to model the distribution of villus, need a parameter to control scale factor of density. Because all section unit use the same scale factor, 
so the villus density of every section unit is proportional with its area. Then, we can still get right distribution style of villus without dividing plant surface grid regularly.

If adopt immediate method to model distribution of villus, need a parameter to control the number of villus on every section surface. In default, the number of villus on the same surface is same. Because of the shape of plant organs are very irregular, so the surface grid of plant organs are constituted by irregular polygons possibly. So, define the villus number on every surface as a function which is proportional to surface area, as shown in Eq.1:

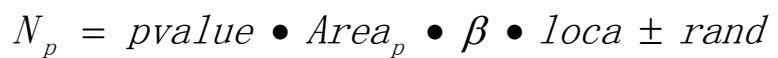

In Eq. $N_{p}$ is the whole number of villus on section surface $P$, Area $_{p}$ is area of $P, \beta$ is control factor, it is used to adjust the inflation of area. loca is the location information of section surface. rand is a random value. loca and rand are selectable, in default, loca is 1, rand is 0 .

\section{Direction}

The direction of villus and section surface should be confirmed when put villus on plant surface. In default, it is vertical. But in fact, villus in not always vertical to the surface of organs, it is direction is uncertain, so, function is used to provide the direction of villus, it is expressed as a random angel.

\section{Length and radium}

The length and radium of villus is a random value in a certain range, and they are different as the different plant, but the radium is decreasing from root to top, that is to say it is a cone.

\section{Examples and discussion}

To test the visualization method which provided by this paper, simulate watermelon blossom and vine villus, Fig. 2 is true photo, and Fig. 3 is the simulation result.

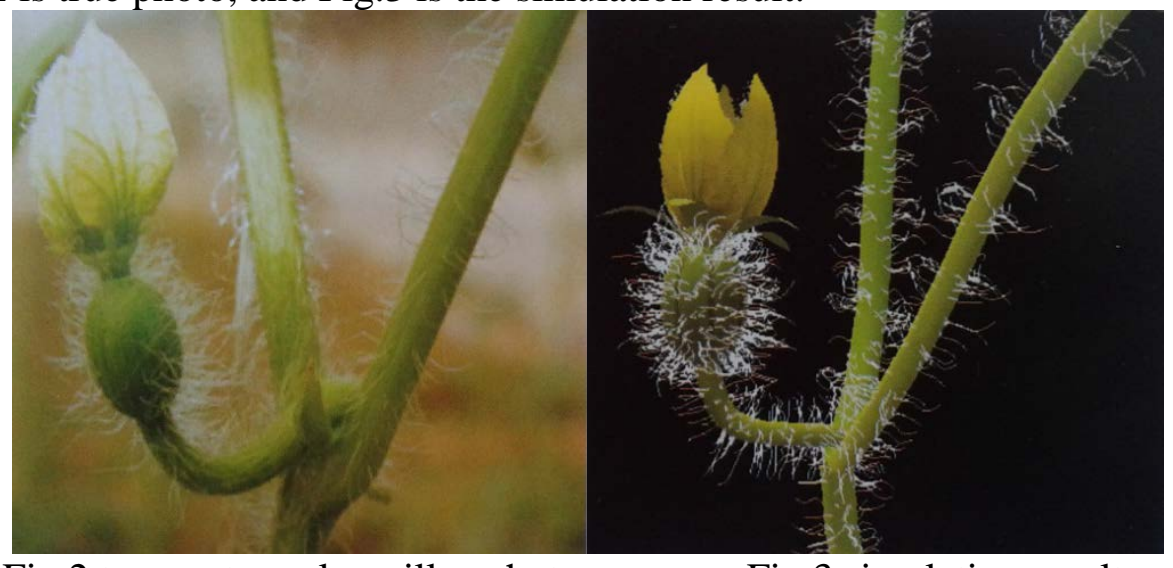

Fig.2 true watermelon villus photo

Fig.3 simulation result

In Fig.3, three different bend villus are used, the direction of villus is random.

Fig. 4 is a true photo of plant tendril and Fig. 5 is the simulation result. Fig. 4 indicate that the length and radium of villus are not even, there is no villus on the end of tendril, so, in the simulation process, control the result in two aspects, one is the location information in longitudinal direction, another is the location information in transverse direction. 




Fig.4 True photo of tendril

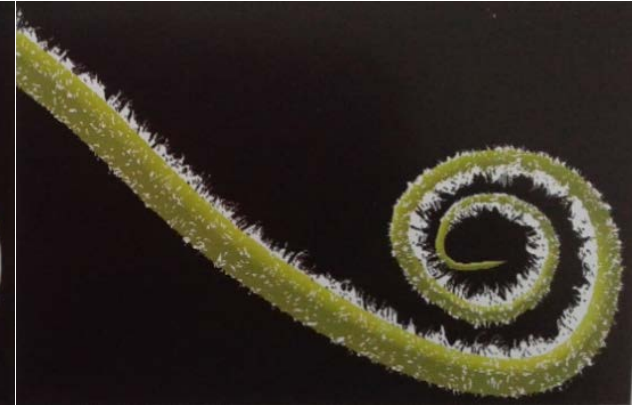

Fig.5 simulation result of tendril

\section{Conclusion}

Villus is an important morphological characteristics of plant, to simulate villus effectively is important to increase the realism feeling of plant simulation model, and, it is also significance in the area of plant species recognition, image synthesis, etc.

The research result indicate that a parametric method can be adopted to construct the villus shape and outward appearance, and the method is easily combined with existing three-dimensional modeling of plants, and the method also considered the specificity of plant villus, using the location information of organ surface as parameter, and add in some random variables to realize its nature effect.

\section{Reference}

[1]Ding Weilong, Hu Chen, Cheng Zhijun, et al. Intelligent modeling method for plant morphology based on gene expression programming[J]. Transactions of the CASE,2013,29(1):134-141.

[2]Wang Dong, Xia Yi, Yin Muyi, et al. Simulation of virtual plant development based on environment sensitivity. Computer Engineering and Applications, 2013, 49(19): 173-176.

[3] Jing Song, Fang Kui, Shen Luming, et al. Three-dimensional structure model of virtual plant growth based on possession in L-system[J]. Journal of Agricultural Mechanization Research, 2013.5(5):20-23.

[4] XIONG Hai-qiao, JIANG Li-hua, LUO Yi-Xian.The plant root growth modeling based on particle system and the physical constraints Acta Agriculture Universities JiangXiENSIS, 2002,(3):24-27

[5] JIANG Li-ping, CHEN Shu-ren. The research progress of virtual plant[J]. Agricultural Mechanization Research, 2006(4):4-6

[6] Tang Wei-dong, LIU Chang-xin, Li Ping-ping, LU Zhang-ping, ZHU Ping. Study on digital construction of plant growth base on information fusion[J].Application Research of Computers, 2010,27(1):174-176 